\title{
Incorrect rotation curve of the Milky Way
}

\author{
Laurent Chemin $^{1,2}$, Florent Renaud ${ }^{3}$, and Caroline Soubiran ${ }^{1,2}$ \\ 1 Univ. Bordeaux, LAB, UMR 5804, 33270 Floirac, France \\ e-mail: astro.chemin@gmail.com \\ 2 CNRS, LAB, UMR 5804, 33270 Floirac, France \\ 3 Department of Physics, University of Surrey, Guildford, GU2 7XH, UK
}

Received 6 March 2015 / Accepted 2 April 2015

\begin{abstract}
In the fundamental quest of the rotation curve of the Milky Way, the tangent-point method has long been the simplest way to infer velocities for the inner low-latitude regions of the Galactic disk from observations of the gas component. In this article, we test the validity of the method on a realistic gas distribution and kinematics of the Milky Way, using a numerical simulation of the Galaxy. We show that the resulting velocity profile strongly deviates from the true rotation curve of the simulation because it overstimates it in the central regions and underestimates it around the bar corotation. In addition, its shape is strongly dependent on the orientation of the stellar bar with respect to the observer. The discrepancies are caused by the highly nonuniform nature of the azimuthal velocity field and by the systematic selection by the tangent-point method of high-velocity gas along the bar and spiral arms, or low-velocity gas in less dense regions. The velocity profile only agrees well with the rotation curve beyond corotation, far from massive asymmetric structures. Therefore the observed velocity profile of the Milky Way inferred by the tangent-point method is expected to be very close to the true Galactic rotation curve for $4.5 \lessgtr R \leq 8 \mathrm{kpc}$. The gaseous curve is flat and consistent with rotation velocities of masers, red clump, and red giants stars measured with VLBI astrometry and infrared spectroscopy for $R \geq 6 \mathrm{kpc}$. Another consequence is that the Galactic velocity profile for $R<4-4.5 \mathrm{kpc}$ is very likely flawed by the nonuniform azimuthal velocities and does not represent the true Galactic rotation curve, but instead local motions. The real shape of the innermost rotation curve is probably shallower than previously thought. Using an incorrect rotation curve has a dramatic effect on the modeling of the mass distribution, in particular for the bulge component, whose derived enclosed mass within the central kpc and scale radius are, respectively, twice and half of the actual values. We therefore strongly argue against using terminal velocities or the velocity curve from the tangent-point method to model the mass distribution of the Milky Way. The quest to determine the innermost rotation curve of the Galaxy remains open.
\end{abstract}

Key words. ISM: kinematics and dynamics - Galaxy: kinematics and dynamics

\section{Introduction}

The quest of the velocity field of the Milky Way has long been a very difficult task. The location of the solar system inside the disk makes it impossible to directly constrain the 3D position and velocity phase space for the whole Galaxy. Most surveys devoted to the chemistry, kinematics, and dynamics of the Galactic $\operatorname{disk}(\mathrm{s})$ are based on optical spectroscopy, which is not appropriate to probe the innermost low-latitude disk regions. It can only be surveyed at infrared, $\mathrm{mm}-$, and $\mathrm{cm}$-wavelengths where obscuration by dust is less problematic. This is one of the objectives of the infrared Sloan Digital Sky Survey III's Apache Point Observatory Galactic Evolution Experiment (APOGEE, Eisenstein et al. 2011). For instance, Bovy et al. (2012) have modeled the kinematics of 3365 red giants and red clump stars from the first year of APOGEE data and found a rotation curve consistent with a power-law model almost perfectly flat within $4 \lesssim R \lesssim 14 \mathrm{kpc}$. Line-of-sight velocities of stars that yield indirect estimates of distances and rotation velocities are not efficient in probing the rotation curve in the innermost regions, however, which would be necessary to establish accurate mass distribution models. The best technique currently in use to do this is probably astrometry of methanol and water masers in high-mass star formation regions with Very Long Baseline Interferometry (VLBI, e.g., Brunthaler et al. 2009; Reid et al. 2009; Asaki et al. 2010; Shiozaki et al. 2011; Honma et al. 2012; Rygl et al. 2012; Xu et al. 2013; Zhang et al. 2014). Preliminary results from these experiments combined within the Bar and Spiral Structure Legacy (BeSSeL) Survey have for the first time directly revealed the spiral structure of the Galaxy and showed an apparently shallow inner rotation curve (Reid et al. 2014). Although very promising, it is too early for BeSSeL to entirely constrain the innermost rotation curve at a statistically significant level, however, since the current number of reliable velocities remains small, with fewer than ten sources inside $R=4 \mathrm{kpc}$.

Instead, $\mathrm{CO}$ and $\mathrm{HI}$ observations of the gaseous interstellar medium have long been used as the reference to establish the inner rotation curve of the Milky Way (Burton \& Gordon 1978; Gunn et al. 1979; Clemens 1985; Fich et al. 1989; McClure-Griffiths \& Dickey 2007; Levine et al. 2008; Sofue et al. 2009; Marasco \& Fraternali 2012). Indirect estimates of rotation velocities and distances are obtained from the tangentpoint (TP) method, which uses terminal line-of-sight velocities (see Sect. 2). Such velocities and the rotation curve inferred from the TP method are the kinematical basis of many Galactic mass models (e.g., Merrifield 1992; Dehnen \& Binney 1998; Kalberla 2003; Famaey \& Binney 2005; Sofue et al. 2009; Sofue 2013), 3D models of the inner neutral atomic and molecular gas disks of the Galaxy (Nakanishi \& Sofue 2003, 2006; Kalberla et al. 2007), or Galactic population synthesis models (Robin et al. 2003).

However, this curve does not perfectly reflect the reality because the gas distribution and kinematics are perturbed by the 


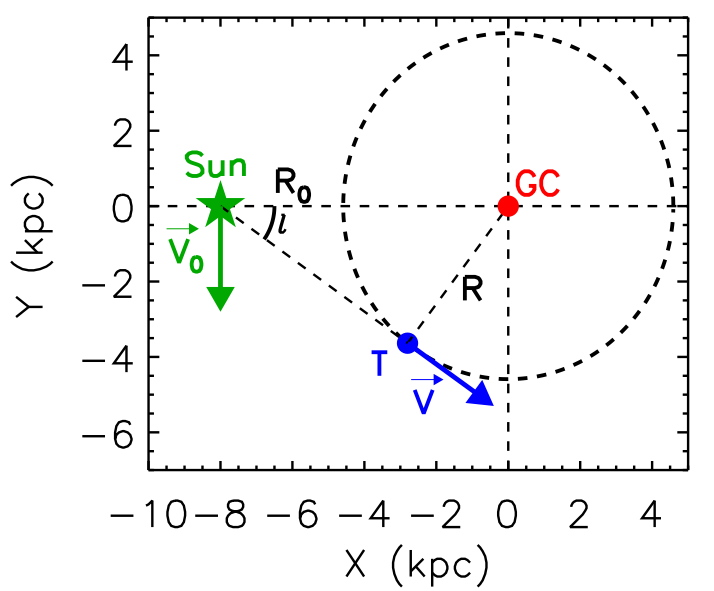

Fig. 1. Geometry of the system: Sun, tangent-point $(T)$, Galactic Center (GC) assumed by the tangent-point method. The Sun is at radius $R_{0}, T$ at radius $R$. The circular velocities are $v_{0}$ at $R_{0}$, and $v$ at $R$, $l$ being the Galactic longitude. We choose here a view similar as in Renaud et al. (2013) where the Northern and Southern Galactic Poles are respectively in front of and behind the reader.

Galactic bar. The effects of the bar on gas terminal velocities and longitude-velocity diagrams in the central regions were first shown by Liszt \& Burton (1980) and Blitz \& Spergel (1991). Numerical simulations of the Galactic interstellar medium have shown the dependency of such diagrams on the position of the observer in the disk (Fux 1999; Englmaier \& Gerhard 1999; Bissantz et al. 2003; Rodriguez-Fernandez \& Combes 2008). As a consequence, most of mass models restricted the analysis to $|l|>15-20^{\circ}$ or $R>0.2 R_{0}$ ( $l$ is the longitude, $R$ is the Galactocentric radius and $R_{0}$ the Galactocentric radius of the Sun), to minimize the perturbing effects from the bar at the center. Although laudable, these arbitrary limits remain questionable because the bar and the spiral structure are expected to have a significant effect over more extended regions.

In this paper we propose to test the capacity of the tangentpoint method in predicting a reliable rotation curve using a hydrodynamical simulation of a Milky Way-like galaxy (Renaud et al. 2013). This simulation sets up a live model of the Galaxy in a self-consistent way, that is, without a fixed pattern speed. The resulting structure and velocity field of the stellar and gaseous components are far from being axisymmetric and circular. In particular, noncircular velocities as high as $200 \mathrm{~km} \mathrm{~s}^{-1}$ are found along the major axis of the bar. We investigate the effect of the nonaxisymmetric perturbations on the shape and amplitude of the inferred curve and on mass models. We analyze the properties of tangent points and constrain the radial range over which the tangent-point method provides a reliable estimate of the actual rotation curve. The basics of the tangent-point method are described in Sect. 2, the confrontation to the gas simulation and a discussion of the limits of the method is provided in Sects. 3 and 4 , and the consequences for mass distribution modeling and for the Galaxy are detailed in Sect. 5.

\section{The tangent-point method applied to the Galaxy}

For any given Galactocentric radius $R$ smaller than the solar radius, there are two line-of-sights at longitude $\pm l$ in the Galactic plane that are tangent to the circle of radius $R$ (Fig. 1). The tangent-point method stipulates that the line-of-sight (1-o-s) velocity $v_{T, \text { los }}$ is an extremum at the position of the tangent point $T$. The Galactocentric radius and circular velocity profile $v(R)$ of

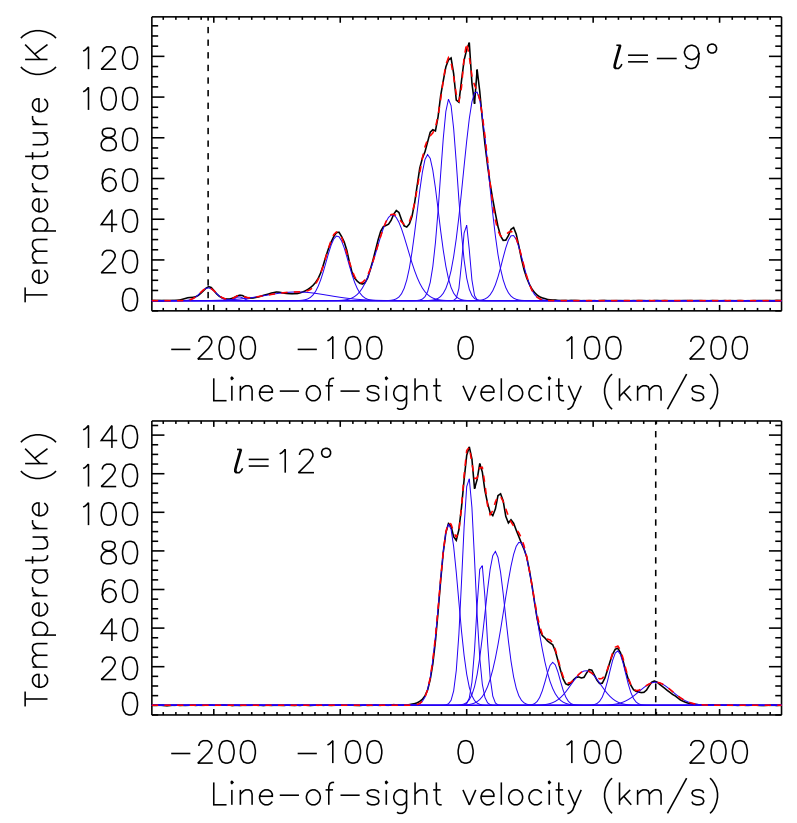

Fig. 2. Examples of spectral decomposition of the Hi profiles at $l=-9^{\circ}$ and $l=12^{\circ}$. The solid black curve shows the observed profile, which is decomposed using multiple Gaussian functions (in blue). The reconstructed profile is shown as a dashed red curve. The vertical dashed line shows the terminal velocity of the peak selected to derive the rotational velocity with Eq. (2).

such material at Galactic latitude $b=0^{\circ}$ are expressed as a function of $l$ and the terminal velocity $v_{T, \text { los }}$ by

$$
\begin{aligned}
R & =R_{0} \sin (l), \\
v(R) & =v_{0} \sin (l)+v_{T, \text { los }},
\end{aligned}
$$

where $v_{0}$ is the circular velocity at the Galactocentric radius $R_{0}$. It is straightforward to estimate the rotation velocity profile from Eq. (2) with mm- or cm-observations by selecting the $\mathrm{HI}$ or CO spectral component whose 1-o-s velocity $v_{T, \text { los }}$ is lowest for $l<0^{\circ}$ and highest for $l>0^{\circ}$.

We have applied the TP method to the $\mathrm{HI}$ Leiden/Argentine/Bonn Survey datacube of the Milky Way (Kalberla et al. 2005). This survey yields the most sensitive all-sky dataset of the neutral atomic gas of the Galaxy at angular and spectral samplings of $0.5^{\circ}$ and $2 \mathrm{~km} \mathrm{~s}^{-1}$. We used $R_{0}=8 \mathrm{kpc}$ and $v_{0}=213 \mathrm{~km} \mathrm{~s}^{-1}$ to facilitate comparison with the numerical simulation (Sect. 3). These are not the IAU values of $8.5 \mathrm{kpc}$ and $220 \mathrm{~km} \mathrm{~s}^{-1}$, but the choice of the fundamental parameters does not affect the result. Hi spectra were decomposed on a base of Gaussian functions, following the method used for the Andromeda galaxy (Chemin et al. 2009). In the example shown in Fig. 2, the spectra were fitted with nine Gaussian profiles. The selected velocity extrema of $\sim-205 \mathrm{~km} \mathrm{~s}^{-1}$ at $l=-9^{\circ}(R=1.25 \mathrm{kpc})$ and $\sim 150 \mathrm{~km} \mathrm{~s}^{-1}$ at $l=12^{\circ}(R=1.66 \mathrm{kpc})$ correspond to velocities of $238 \mathrm{~km} \mathrm{~s}^{-1}$ and $194 \mathrm{~km} \mathrm{~s}^{-1}$, respectively.

By repeating this exercise for $-90^{\circ}<l<90^{\circ}$, we obtained the velocity curves shown in Fig. 3, which agree very well with many other curves using the same method from $\mathrm{CO}$ or Hi data (e.g., Burton \& Gordon 1978; Clemens 1985; Sofue et al. 2009; Marasco \& Fraternali 2012). The steep profile in the center is clearly visible, as is the peak at $\sim 260 \mathrm{~km} \mathrm{~s}^{-1}$ at $500 \mathrm{pc}$, the smooth decrease to $\sim 200 \mathrm{~km} \mathrm{~s}^{-1}$, the increase beyond $R \sim$ $3.5 \mathrm{kpc}$, and flat profiles at large radii. The axisymmetry between the two quadrants is quite remarkable for $R>3.5 \mathrm{kpc}$. 


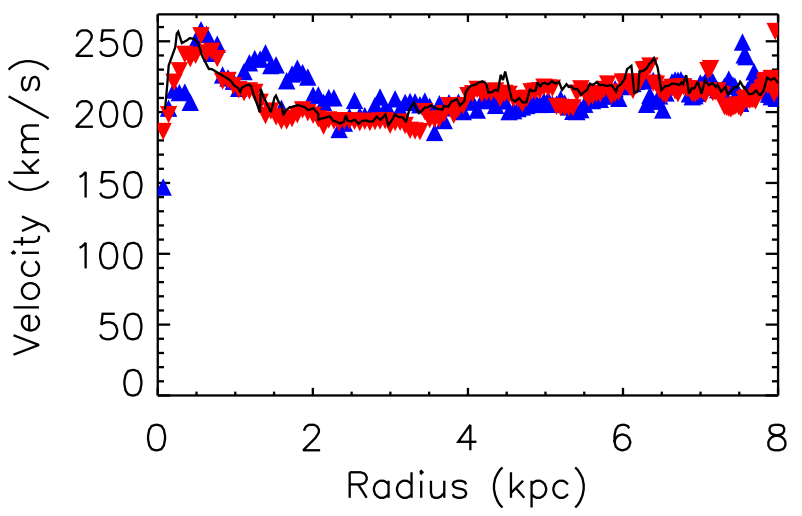

Fig. 3. Velocity profile of the Milky Way inferred with the tangent-point method applied to the HI LAB datacube of Kalberla et al. (2005), assuming $R_{0}=8 \mathrm{kpc}$ and $v_{0}=213 \mathrm{~km} \mathrm{~s}^{-1}$. Blue upward triangles are for $-90^{\circ} \leq l<0^{\circ}$, red downward triangles for $0^{\circ} \leq l \leq 90^{\circ}$. The solid line is the composite curve from Sofue et al. (2009) normalized at our value of $v_{0}$.

The disk at $l<0^{\circ}$ appears to rotate faster than at positive longitudes by $\sim 17 \mathrm{~km} \mathrm{~s}^{-1}$ on average for $1<R<3.5 \mathrm{kpc}$. Note in particular the bump at $R \sim 1.5 \mathrm{kpc}$ for $l<0^{\circ}$ that is not measured for $l>0^{\circ}$. It has often been argued that differences in the central regions are signatures of perturbations, like the bar and/or the spiral structure. This is a possible explanation, but we show in Sect. 3.2 that a velocity difference is not the best criterion to detect signatures of perturbations. Notice finally that the composite curve of Sofue et al. (2009) is more consistent with our velocities for $l>0^{\circ}$ than for $l<0^{\circ}$.

\section{The tangent-point method to the test of numerical simulations}

\subsection{Mock gas datacubes}

To quantify to which extent the TP method gives a reasonable rotation curve, we applied it to a simulation of the Milky Way, for which the rotation curve can be obtained directly from the velocity field. We used the simulation of Renaud et al. (2013), which reproduces the main structures (bar, spirals) of the Galaxy both in terms of morphology and kinematics. We produced mock observations by computing longitude, latitude, and line-of-sight velocity datacubes for an arbitrary position of the Sun along the $R_{0}=8 \mathrm{kpc}$ circle. Varying the position of the Sun allows us to monitor the effects of the bar orientation on the results from the TP method. In our fiducial case, the bar major axis yields an orientation of $23^{\circ}$ with respect to the direction of the Galactic center. This angle setup matches the real Galaxy best (Renaud et al. 2013). The covered range of bar orientations is $0^{\circ}$ (Sun aligned with the bar) to $90^{\circ}$ (Sun - Galactic center axis perpendicular to the bar major axis). It allows us to test, for instance, whether the bar viewing angle of $\sim 45^{\circ}$ inferred from some IR observations (Hammersley et al. 2000; Benjamin et al. 2005) would yield results different from our fiducial case. The velocities are computed from the gaseous (atomic and molecular) component of the model. The spatial and spectral samplings of these mock datacubes are $2^{\circ}$ for $(l, b)$ and $3 \mathrm{~km} \mathrm{~s}^{-1}$ for 1-o-s velocity. These samplings are smaller than for the Hi observation, implying smoother velocity profiles, but this does not affect the result of this work. A datacube was also generated using an earlier epoch of the simulation. It corresponds to an

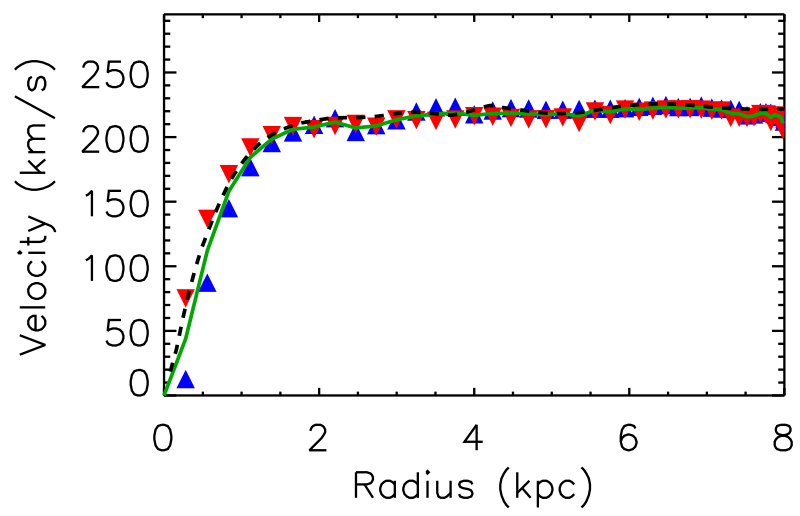

Fig. 4. Rotation velocity profile of the simulated disk inferred by the tangent-point method for the axisymmetric disk (before the formation of the bar and spiral arms). Colored symbols are the same as in Fig. 3. The solid line is the average curve from the two halves. The dashed line is the true rotation curve of the simulated disk.

axisymmetric disk, that is, a reference case before the formation of the bar and the spiral arms.

\subsection{Velocity profiles}

Figure 4 shows the resulting velocity profile for the reference datacube of the axisymmetric disk. The velocity increases within the inner $1.5 \mathrm{kpc}$ and then remains constant with radius. The velocity curve averaged over positive and negative longitudes is exactly the one inferred from averaging the azimuthal velocity field of gas cells from the simulation at every radii. This implies that there are no systematics in our approach and that the TP method can yield a velocity profile that excellently agrees with the true rotation curve if the disk remains nearly axisymmetric.

Using the snapshot that best matches the observations, that is, after the formation of the bar and the spiral arms, Fig. 5 shows the velocity profiles for a selection of several bar viewing angles. The shape and maximum amplitude of the velocity profile strongly depend on the bar viewing angle. The more aligned the reference point with the bar major axis, the higher the velocity peak at small radius. Moreover, the slope of the velocity profile in the central region decreases with increasing viewing angle. Configurations with angles between $0^{\circ}$ and $23^{\circ}$ all show a decrease of velocity from $R=0.5-1 \mathrm{kpc}$ to $R \sim 4 \mathrm{kpc}$, while the velocity increases up to $R=3-3.5 \mathrm{kpc}$ for larger viewing angles. The velocity peak is thus offset for large viewing angles. With the exception of the profile for an angle of $90^{\circ}$ that continuously overestimates the true values beyond $3.5 \mathrm{kpc}$, most configurations yield velocities that agree fairly well with the correct rotation curve for $R>4.5 \mathrm{kpc}$. In addition, the velocity is constant beyond $5.5 \mathrm{kpc}$. A common feature to most velocity profiles is the inflection of the curve past the bar corotation $\left(R_{\mathrm{c}}=3.6 \mathrm{kpc}\right)$.

The velocity difference between the two quadrants strongly varies with the bar viewing angle. For instance, the two quadrants yield very similar velocity curves at every radius for configurations of $15^{\circ}$ or $45^{\circ}$, while larger differences are found at $0^{\circ}$ and $67^{\circ}$. The asymmetry is also stronger for $R<4.5 \mathrm{kpc}$ at $23^{\circ}$ and $67^{\circ}$. The bar and spiral arms cannot be detected when viewed at $15^{\circ}$ and $45^{\circ}$, with the assumption that differences between the two quadrants trace nonaxisymmetric structures. Similarly, it is difficult to reconcile the asymmetry at $0^{\circ}$ and $67^{\circ}$ at large radii where the perturbations are weaker with the axisymmetry at similar radii for all other configurations. Differences between the two quadrants thus cannot be the sole 

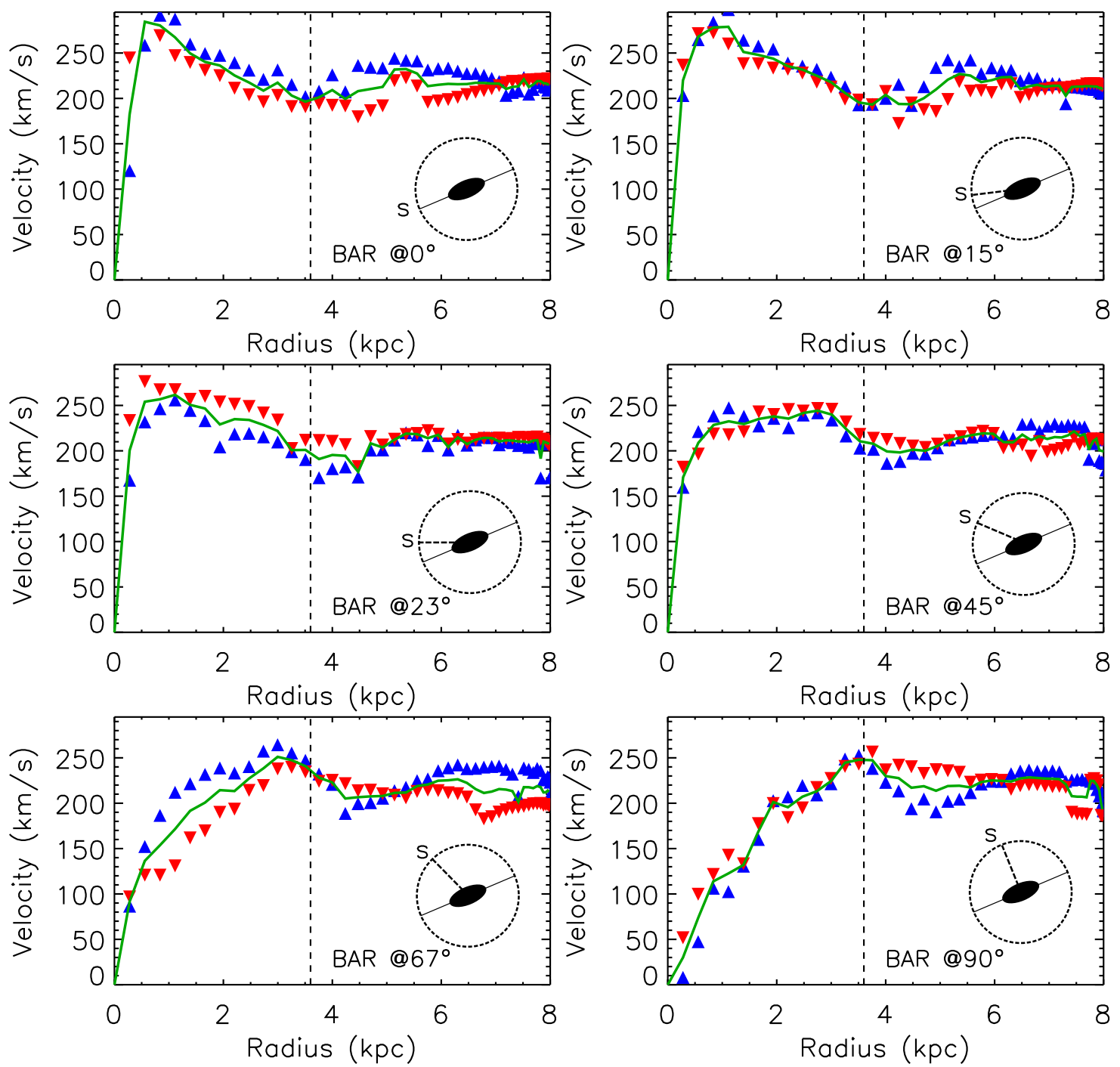

Fig. 5. Rotation velocity profile of the simulated disk for several viewing angles of the bar. The vertical dashed line is the radius of the bar corotation, as computed in Renaud et al. (2013).

criterion to attest or reject the presence of nonaxisymmetric structures like the bar, or assess its strength.

The comparison between the rotation curve of the mock Galactic disk and the velocity profiles from the TP method all given at $\left(R_{0}, v_{0}\right)=\left(8 \mathrm{kpc}, 213 \mathrm{~km} \mathrm{~s}^{-1}\right)$ is very instructive (Fig. 6). The tangent-point method rarely gives a velocity profile that agrees with the rotation curve for $R<4.5 \mathrm{kpc}$. The profiles for bar viewing angles $\lesssim 45^{\circ}$ overestimate the rotation curve inside $R=2.5-3 \mathrm{kpc}$ and then underestimate it past this radius. Almost all profiles are consistent with the true rotation beyond $R=4.5 \mathrm{kpc}$. The extreme viewing angle of $90^{\circ}$ is the only model that uniformly overstimates the rotation curve at these radii, while it understimates it in the inner regions. To illustrate the significant discrepancy at small radius, the difference of velocities for the fiducial bar orientation of $23^{\circ}$ is $100 \%$ of the true velocity at $R=0.5 \mathrm{kpc}, \sim 50 \%$ at $R=1 \mathrm{kpc}$, and $18 \%$ at $R=3.7 \mathrm{kpc}$, close to the bar corotation.

Figure 6 also shows the comparison with the observational Hi data. The agreement is very good at $R>4-4.5 \mathrm{kpc}$ for most of bar orientations. Bar viewing angles of $23^{\circ}$ and $30^{\circ}$ are the models that best reproduce the central slope and the amplitude of the peak at $R=0.5 \mathrm{kpc}$. Smaller angles overestimate the peak, while larger angles underestimate it. Angles of $67^{\circ}$ and $90^{\circ}$ are

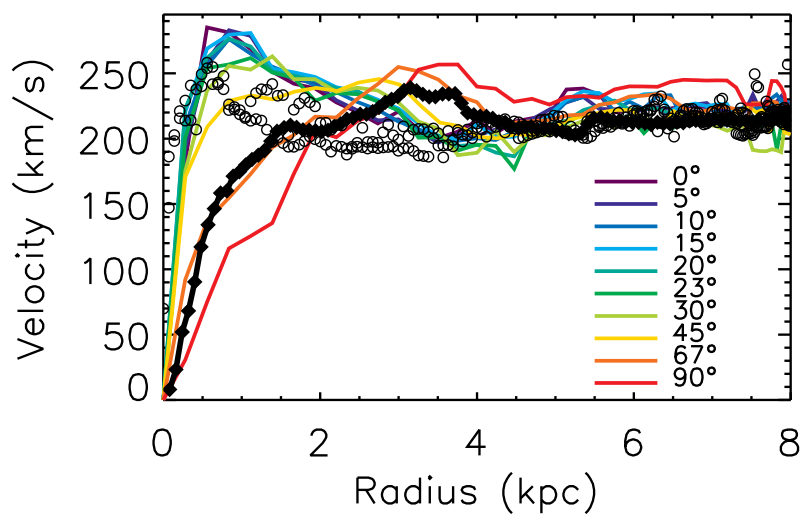

Fig. 6. Comparison of the true rotation curve of the simulated disk (filled symbols) with the velocity profiles inferred by the tangent-point method (colored lines). The viewing angle of the bar is indicated for each velocity profile. The open circles represent the velocities from the Hi observations of the Milky Way.

the least compatible models with the observed velocities. On average, the shape and amplitude of velocities in the fourth quadrant $\left(l<0^{\circ}\right.$, upper part of the observational points) are more 

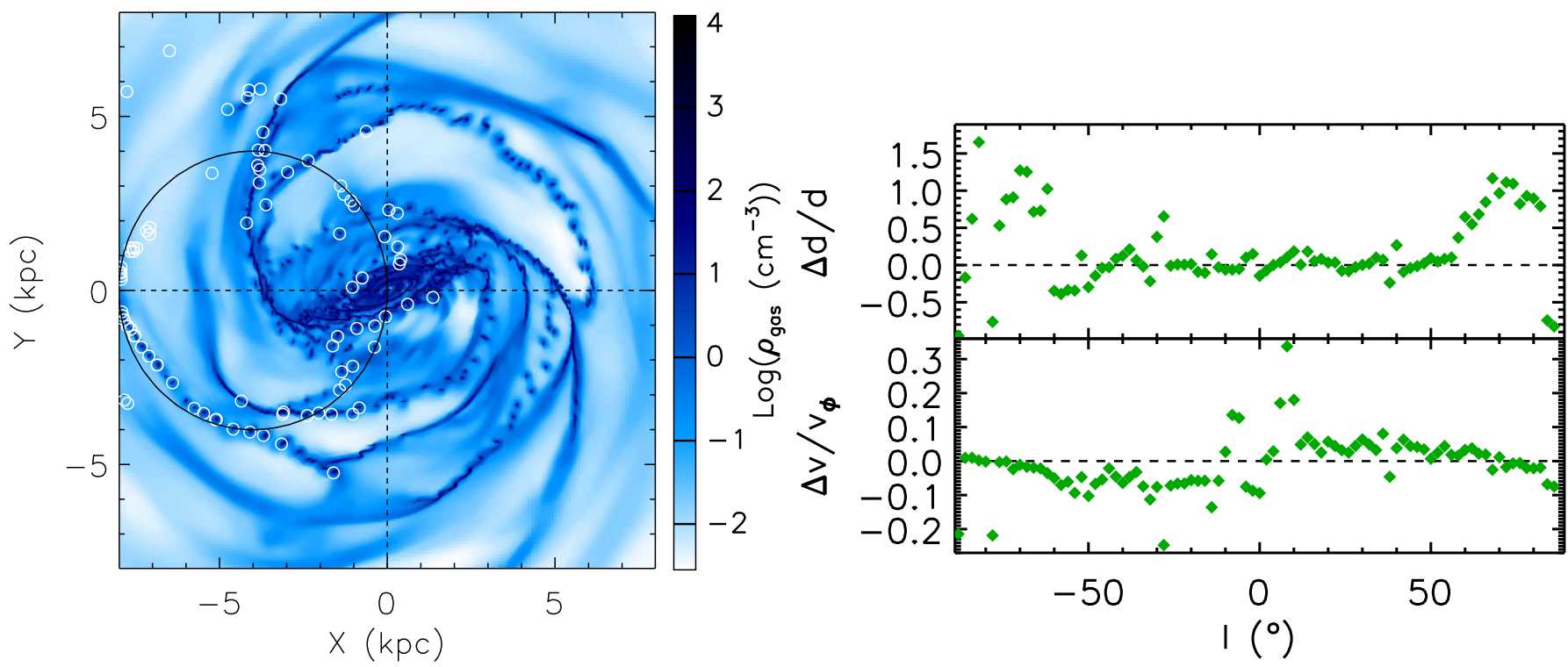

Fig. 7. Left: gas density map showing the locations of terminal l-o-s velocity points (white circles). The black circle indicates the positions of the tangent points. The reference position is $(x, y)=(-8,0) \mathrm{kpc}$. Right: comparison of the distance (top) and velocity $($ bottom) of the tangent points with the true distance and velocity of the terminal velocity points. $\Delta d=d_{\mathrm{T}}-d$ where $d_{\mathrm{T}}=\left(R_{0}^{2}-R^{2}\right)^{1 / 2}$ is the distance to tangent points and $d$ the true distance to terminal velocity points. $\Delta v=v(R)-v_{\phi}$, where $v(R)$ is from Eq. (2) and $v_{\phi}$ is the true azimuthal velocity of terminal velocity points.

consistent with the models than in the first quadrant (lower part of the points).

\section{Limits of the tangent-point method}

\subsection{Properties of tangent points and terminal velocity points}

Although the tangent-point method is appropriate for the early stage snapshot (Fig. 4), its validity is clearly questionable for the asymmetric disk. To address this problem, we analyzed the characteristics of the tangent points and terminal velocity points. We based the analysis only on the fiducial case (bar at $23^{\circ}$ ) because it represents the real Galaxy best (Renaud et al. 2013).

We first address the question whether tangent points are actual terminal velocity points. This goal can be achieved by comparing the tangent points with the positions and azimuthal velocities of the terminal velocity points. We find the gas at the origin of the terminal velocities by searching the clouds whose 1-o-s velocity is within $\pm 5 \mathrm{~km} \mathrm{~s}^{-1}$ from the terminal velocity. This velocity range corresponds to a window of $5 \sigma$, where $\sigma$ is the spectral sampling of the mock datacube. Since more than one cloud may contribute to shape the emission profile around the terminal velocity, we expect to find a few lines of sight that harbor several clouds within the search window. In this case, we kept the cloud with the brightest ${ }^{1}$ contribution to the emission profile because it represents that population of clouds best. Note that a wider search window only marginally changes the identified clouds.

Figure 7 shows the corresponding locations of the terminal velocity points. These points are almost exclusively clouds lying along the spiral arms and the bar. A few positions are not associated with a specific cloud, but correspond to less dense gas in inter-arm regions (e.g., $x, y \sim-1.3,2.5$ ), or along a more diffuse spiral structure (e.g., $x<-6, y>0$ ). However, it is striking that terminal velocity points that coincide perfectly with the tangent points whose locations are delineated by a circle are rare.

\footnotetext{
1 We roughly estimate the brightness for the simulation as the gas density divided by the square of the distance to the Sun.
}

In particular, the pitch angle of the spiral arms passing at the Sun position causes the tangent-point method to systematically overestimate the true distance at high longitudes.

Figure 7 shows the errors on the true distance to the terminal velocity points $(d)$ when assuming that the distance is that of the tangent points $d_{\mathrm{T}}=\left(R_{0}^{2}-R^{2}\right)^{1 / 2}$ (see Fig. 1). On average, the difference in distance is $13 \%$ for $-55^{\circ}<l<0^{\circ}$ and $8 \%$ for $0^{\circ}<l<55^{\circ}$. That ratio increases at $|l|>55^{\circ}$, where it is $\sim 86 \%$ on average. Therefore, the closer to the Sun the tangent point, the less accurate the distance $d_{\mathrm{T}}$. Figure 7 also shows the difference between the velocity of the tangent point as given in Eq. (2) and the local azimuthal velocity at the position of the terminal velocity point. The tangent-point velocity underestimates (overstimates) the azimuthal velocity at negative (positive) longitudes. The difference increases toward low longitudes. On average, a difference of $7 \%$ is measured for $-50^{\circ}<l<-10^{\circ}$ relative to the local azimuthal velocity; this is $4 \%$ for $10^{\circ}<l<50^{\circ}$. The error remains relatively constant within this longitude range. It is negligible at high (absolute) longitudes and can be larger than $15 \%$ in the inner $|l|<10^{\circ}$. We conclude that the tangent points and terminal velocity points are thus quite similar for intermediate longitudes, but are certainly not the same at the lowest and highest longitudes, or at particular outlier directions. The effect of the significant difference of position relative to the true position at large $l$ is negligible for velocities, however, since the rotation velocity does not vary significantly at large radii.

Similarly, we analyzed the differences between the tangentpoint velocity and the local azimuthal velocity at the position of the tangent-points. Here we again find a strong dependency on longitude, but the scatter is about five times larger than what is shown in Fig. 7 for terminal velocity points. In other words, a tangent-point velocity is surprisingly less consistent with its local azimuthal velocity than with the azimuthal velocity at the position of the terminal velocity point.

Interestingly, we note that velocity profiles based either on the local azimuthal velocity (and position) at the terminal velocity points or on the local azimuthal velocity at the tangent-points 
differ significantly from the rotation curve for $R<5 \mathrm{kpc}$. Their shape remains similar to $v(R)$ of Figs. 5 and 6, again with velocities overestimated in the inner regions, and is underestimated around the bar corotation.

The distance errors have previously been estimated by Gómez (2006), but from more idealized gas flow simulations than the one we used, for a disk perturbed by a $m=2$ spiral within the fixed potential of Dehnen \& Binney (1998). They have found mean errors lower than $0.5 \mathrm{kpc}$, and larger errors in the spiral arms. Our result is thus quite consistent with their estimate, on average.

\subsection{Origin of the discrepancy with the rotation curve}

We have shown that Eqs. (1) and (2) are rough - and sometimes incorrect - approximations of the local positions and azimuthal velocities of terminal velocity points and are even poorer estimates of the local azimuthal velocities at the coordinates of tangent points. However, the position and velocity errors are not issues as such. Indeed, it remains difficult to recover the shape of the rotation curve of the mock disk, irrespective of the nature of the points or velocities chosen to calculate the velocity profile. At the same time, we have shown that the bar orientation with respect to an observer strongly affects the shape of the inferred velocity profile. Ignoring the effect of the bar therefore renders the TP method useless in regions where the bar dynamics dominates the motions, and one may thus accordingly question its validity in estimating the true rotation curve of a barred spiral galaxy like the Milky Way.

The discrepancy with the rotation curve is not directly linked to the tangent-point method itself. It is a combination of several elements: the asymmetric nature of the disk, the nonazimuthal dependency of any rotation curve, and the location of the Sun. It is indeed the structure of the velocity field itself that causes the peculiar shape of the velocity profile: the tangential velocities of gas are highly nonuniform. Perfect axisymmetry and uniform tangential motions do not exist. This is evidenced by the strong dependency of tangential velocities on azimuth (Fig. 8, at $R=1 \mathrm{kpc}$, e.g.). The axisymmetric value is $\sim 175 \mathrm{~km} \mathrm{~s}^{-1}$, whereas the bulk of gas rotates with velocities ranging from $100 \mathrm{~km} \mathrm{~s}^{-1}$ to $290 \mathrm{~km} \mathrm{~s}^{-1}$, with a few points down to $50 \mathrm{~km} \mathrm{~s}^{-1}$. The rotation curve smears out such nonuniform tangential motions because a rotation curve only depends on $R$. With the tangent-point method, the velocity field is unavoidably restricted to two velocities at each radius, arising from the two quadrants. We showed that this is sufficient to derive the rotation curve for the early stage in the simulation since the tangential velocity is uniform at that point, the disk being almost perfectly axisymmetric. However, this is not the case anymore for the barred and spiral configuration. The location of the Sun reference point (thus the viewing angle of the bar) causes the two velocities to coincide with high-velocity clouds along the bar and in the spiral arms, or low-velocity gas in lower density regions. They can therefore only be representative of local nonuniform tangential motions, not of the global rotation curve. Figure 8 illustrates this argument at $R=1 \mathrm{kpc}$, where the velocities of the two terminal-velocity clouds coincide with the upper envelop of the curve. Larger (smaller) bar viewing angles than $23^{\circ}$ would shift the triangles to the left (right) along the curve. At viewing angles of $\sim 67^{\circ}$, the velocities would be almost coincident with the horizontal line (azimuths of $\sim 50^{\circ}$ and $230^{\circ}$ ), and the rotation curve would be correctly recovered (see also Fig. 6).

Confirmation of the local motions affected by the bar at low radius is also seen in the radial velocity $v_{R}$ profile, where $v_{R}$ are
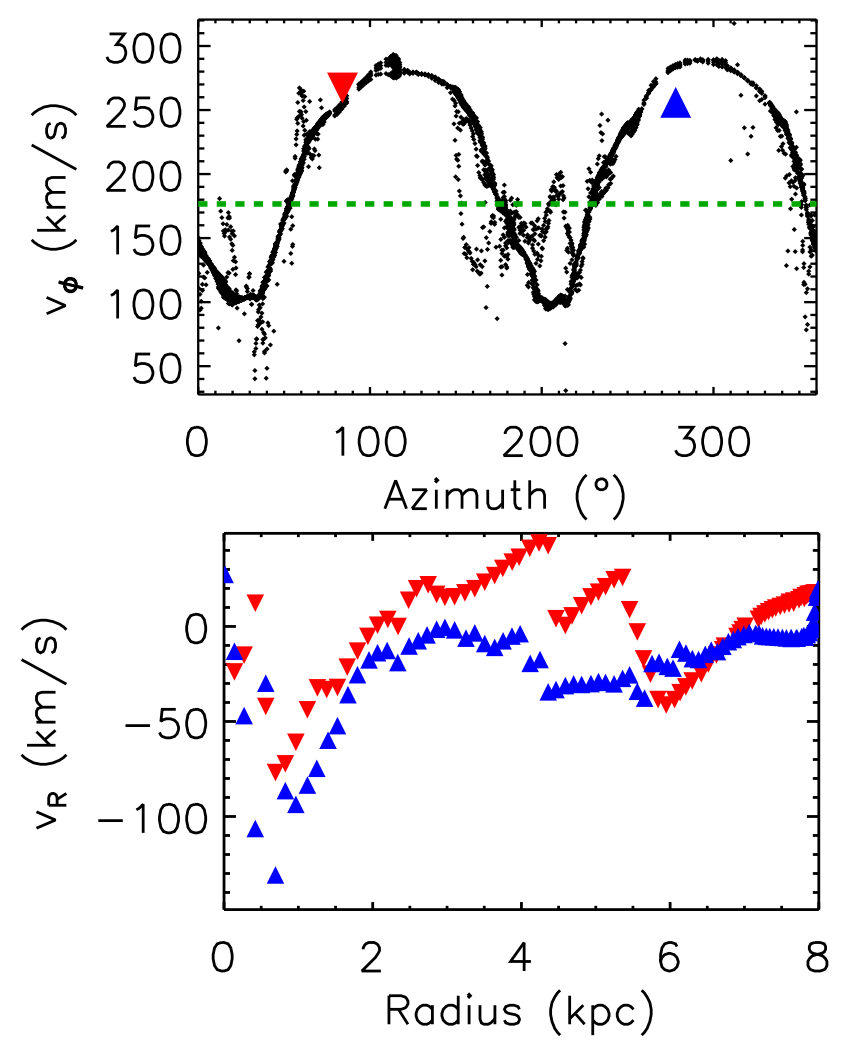

Fig. 8. Top: azimuthal profile of tangential velocity at $R=1 \mathrm{kpc}$. Colored symbols are the velocities inferred by the tangent-point method at a similar radius for the two quadrants. The horizontal dashed line is the azimuthal average of the velocity profile, hence the velocity of the rotation curve at $R=1 \mathrm{kpc}$. The zero azimuth corresponds to the direction of the Sun. Bottom: profile of radial velocity $v_{R}$, locally given at the positions of the tangent points. Each value is not an azimuthal average from the radial velocity field at each radius. Symbols are the same as in Fig. 3.

given here at the positions of the tangent-points (Fig. 8, bottom panel). Significant noncircular motions down, for example, to $\sim-100 \mathrm{~km} \mathrm{~s}^{-1}$ are detected exactly where the velocity profile of Fig. 6 differs the most from the rotation curve, as well as at larger radius. This is not fortuitous, as the asymmetries affect both the circular and noncircular motions.

\section{Discussion}

\subsection{Implication for the mass distribution}

A straightforward implication of using the incorrect rotation curve based on the tangent-point measurements is the incorrect modeling of the mass distribution at the center of the galaxy. Many mass models of the Milky Way invoke a dominant contribution of the stellar bulge in the central regions to explain the steepness and the peak of the profile seen in Fig. 3 (e.g., Merrifield 1992; Sofue et al. 2009; Sofue 2013). We proceed similarly with the simulation using the incorrect rotation curve from the fiducial case and fit a bulge that best reproduces the velocities for $R<0.8 \mathrm{kpc}$ (Fig. 9).

The differences between the contributions from true bulge of the simulation and the incorrect fitted bulge are shown in Fig. 9. The true bulge contribution is not a fit to the true rotation curve, but comes directly from the identification of stellar bulge particles in the simulation. 


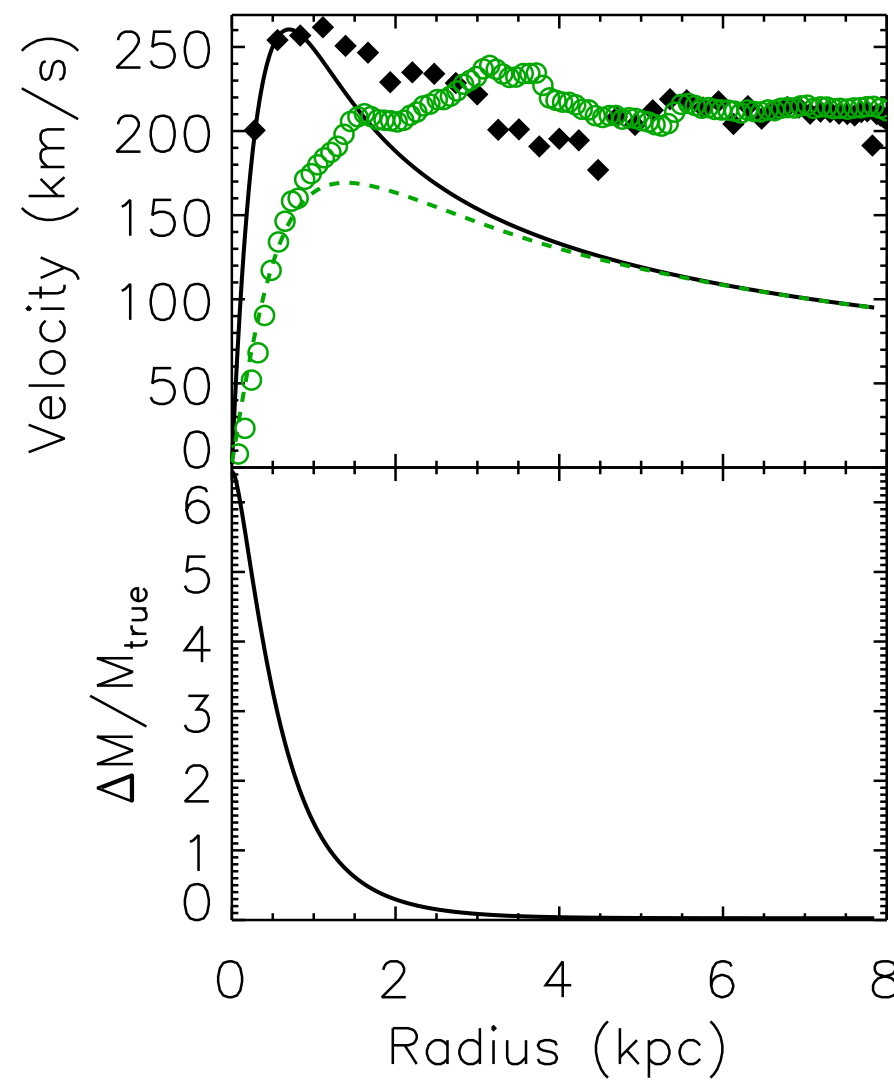

Fig. 9. Top: mass distribution models with the true and incorrect rotation curve of the simulated galaxy. Open symbols and the green dashed line denote the true rotation curve and the bulge contribution, filled symbols and a solid line represent the incorrect rotation curve and bulge contribution. For clarity, the remaining contribution from the gaseous and stellar disks plus dark matter is not shown. Bottom: error on the integrated mass of the bulge. $\Delta M=M_{\text {inc }}-M_{\text {true }}$, where $M_{\text {inc }}$ is the mass deduced from the incorrect bulge, and $M_{\text {true }}$ the true bulge mass.

The true and false bulges are modeled by a spherical density profile given by

$\rho(r)=\rho_{-2} \exp \left(-2 n\left(\left(r / r_{-2}\right)^{1 / n}-1\right)\right)$.

It is equivalent to an Einasto model (Navarro et al. 2004), where $r_{-2}$ is the scale radius at which the density profile has a logarithmic slope of $-2, \rho_{-2}$ is the scale density at that radius, and $n$ a dimensionless index describing the shape of $\rho(r)$. At fixed scale density and radius, the smaller the index, the shallower the density profile in the center (Chemin et al. 2011).

The parameters we found for the incorrect bulge are $\rho_{-2}=$ $10.15 \times 10^{-1} M_{\odot} \mathrm{pc}^{-3}, r_{-2}=0.42 \mathrm{kpc}$, and $n=0.95$. The velocity peak of the incorrect bulge is $260 \mathrm{~km} \mathrm{~s}^{-1}$ at $R=0.7 \mathrm{kpc}$, thus $54 \%$ higher than the true bulge peak, and at a radius twice smaller. Note that the velocity shape and amplitude of that incorrect bulge are comparable with those seen in mass models of the Milky Way (Sofue et al. 2009). The Einasto parameters of the true bulge are $\rho_{-2}=9.46 \times 10^{-1} M_{\odot} \mathrm{pc}^{-3}, r_{-2}=0.87 \mathrm{kpc}$, and $n=1.14$. The incorrect bulge is thus about twice smaller and slightly denser than the true bulge. As expected, its mass distribution is therefore more concentrated than the true one for a similar enclosed masses $\left(\sim 1.6 \times 10^{10} M_{\odot}\right.$ for the true bulge and $\sim 1.7 \times 10^{10} M_{\odot}$ for the incorrect bulge inside $R=8 \mathrm{kpc}$ ). The effect of this higher mass concentration on the enclosed bulge mass is shown in the bottom panel of Fig. 9. As rule of thumb, the considerable error on the enclosed mass is $\sim 330 \%$ of the true mass inside $R=0.5 \mathrm{kpc}$, or $\sim 140 \%$ inside $R=1 \mathrm{kpc}$, the incorrect enclosed bulge mass being 4.3 and 2.4 times higher than the true one inside these radii. Note that the stellar disk plus dark matter halo contribution remains similar in both mass models.

\subsection{Implications for the Milky Way}

In view of the results, the tangent-point method and terminal velocities very probably yield an incorrect rotation curve of the Milky Way inside $R=4-4.5 \mathrm{kpc}$. The steep central gradient, the peak at $R=0.5 \mathrm{kpc}$, and the smooth decrease to $R=3.5 \mathrm{kpc}$ reflect the highly nonuniform nature of tangential velocities, where gas locally orbits faster or slower inside the Galactic bar, the spiral arms, and the interarm regions than the average at the same radii. These local features cannot be representative of the true inner rotation curve of the Galaxy. Beyond $R=4-4.5 \mathrm{kpc}$ $\left(R / R_{0}>0.5-0.56\right)$, it is reasonable to assume that the Galactic rotation curve is consistent with the velocity profile obtained by the tangent-point method, unless stronger asymmetries than expected by the simulation exist at these radii in the Galactic disk. As a consequence, mass distribution models of the Milky Way based on terminal velocities in the inner regions are unavoidably flawed. We estimate that the Galactic bulge might be less concentrated than currently thought, with a characteristic size of $\sim 1 \mathrm{kpc}$, thus about two times larger than inferred by mass models based on terminal velocities.

Furthermore, the tangent-point method gives rise to an inner steep velocity profile and a peak under particular circumstances, when the bar is viewed with angles $<45^{\circ}$. The central velocity peak is always overestimated at viewing angles $\$ 20^{\circ}$. Mock velocity profiles with bar orientations of $23^{\circ}$ and $30^{\circ}$ are the models that agree best with the observational velocities. Galactic bar viewing angles of $\sim 23-30^{\circ}$ are thus favored by our analysis. This agrees with many observational or numerical studies (Rodriguez-Fernandez \& Combes 2008; Wegg \& Gerhard 2013; Renaud et al. 2013), but disagrees with the observational value of $\sim 44^{\circ}$ found by Hammersley et al. (2000) and Benjamin et al. (2005). In addition, the inflection of the observed velocity curve at $R=3.5 \pm 0.3 \mathrm{kpc}$ probably marks the position of the corotation of the Galactic bar.

We can deduce a hypothetical, but more realistic, Galactic rotation curve by naively applying a correction factor of $\left(\frac{v(R)}{v_{\text {true }}}\right)_{\text {sim }}$ to the observed profile, which we deduce from the simulation (see Sect. 3.2 and Fig. 9). This first-order approach assumes that the effects inducing errors in the estimate of the rotation curve from the TP method in the simulations are the same as in the real Galaxy. We have no direct way to verify the validity of this assumption and thus, the following hypothetical curve should be considered with caution. It gives for instance velocities that are 2.2, 1.5 and 1.1 times lower than expected by the TP method at $R=0.5,1$, and $2 \mathrm{kpc}$, respectively, and about 1.2 times higher at $R=3.5 \mathrm{kpc}$ (for $v_{0}=213 \mathrm{~km} \mathrm{~s}^{-1}$ ).

Figure 10 compares individual rotation velocities from BeSSeL, the astrometric survey of methanol and water masers in high-mass star-forming regions (Reid et al. 2009, 2014), and the $R \geq 4 \mathrm{kpc}$ stellar rotation curve from APOGEE (Bovy et al. 2012), with our hypothetical gaseous rotation curve. Both stellar and gaseous velocities were rescaled here using $v_{0}=218 \mathrm{~km} \mathrm{~s}^{-1}$, as given in Bovy et al. (2012). The velocity uncertainty for our gaseous rotation curve takes into account the formal error from the Gaussian fits of terminal velocities $\left(<1 \mathrm{~km} \mathrm{~s}^{-1}\right)$, and the systematic errors from (i) the mean velocity difference between 


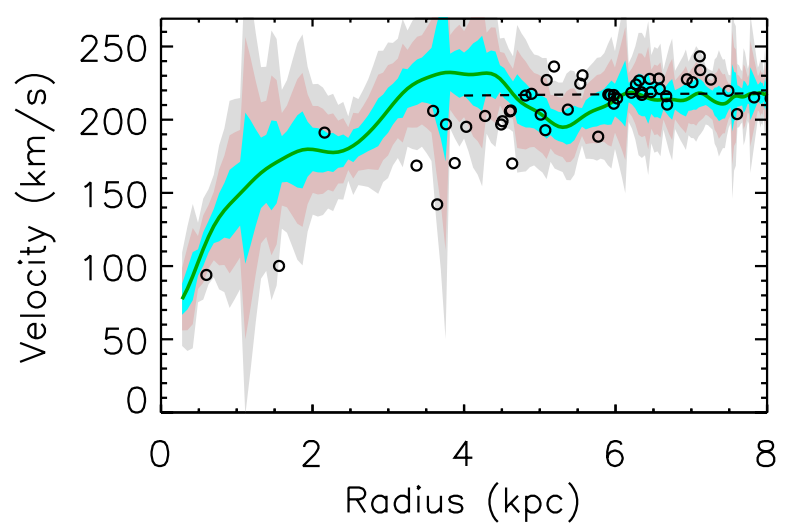

Fig. 10. Hypothetical gaseous rotation curve of the Milky Way (solid line) after correcting for the initial velocity profile of Fig. 3 . The cyan, pink, and gray shaded areas are the 1,2 , and $3 \sigma$ confidence levels. Open circles are rotation velocities of masers from Reid et al. (2014). The dashed line is the power-law rotation curve model of the Galaxy from Bovy et al. (2012).

profiles from simulated datacubes with bar orientations of $23^{\circ}$ and $30^{\circ}\left(5 \mathrm{~km} \mathrm{~s}^{-1}\right)$; (ii) the velocity difference $\Delta v=v(R)-v_{\phi}$ implied by the use of TP method velocities instead of true azimuthal values; and (iii) the velocity difference from both quadrants. These two latter systematic errors depend on the longitude, as seen in Figs. 3 and 7 (right panel). First, it is interesting to note that all rotation curves are consistent for $R>4.5 \mathrm{kpc}$, which clearly shows that the Milky Way rotation curve is consistent with a plateau at $R>6 \mathrm{kpc}$. Secondly, our hypothetical rotation curve tends to overestimate the BeSSeL velocities at $3.5<R<4.5 \mathrm{kpc}$, though remaining in agreement within the $3 \sigma$ confidence level. This is probably due to systematic effects inherent to the construction of our hypothetical curve, that is, to the TP method and the simulation themselves. Note, however, that depending on the location of the masers down- or upstream of the peak densities inside the spiral arms, velocities from BeSSeL may not coincide perfectly with the mean rotation velocities either. They might trace faster or slower local motions, exactly like those seen for individual gas clouds selected by the TP method (Fig 8; see also Fig. 4 in Kawata et al. 2014). It appears essential here that the inner Galactic velocity field has to be covered as uniformly as possible in radius and azimuthal angle to obtain the most accurate inner rotation curve, similarly as is routinely done for nearby galaxies with 3D spectroscopy or radio interferometry (Emsellem et al. 2004; Chemin et al. 2006; de Blok et al. 2008; García-Lorenzo et al. 2015). This would require observations of other tracers than masers in more diffuse regions outside the bar and the arms. This is not yet possible for VLBI, however.

A final consequence of the analysis is that rotation curves of edge-on galaxies based on envelope-tracing of Hi terminal velocities (e.g., Swaters et al. 1997; Kregel \& van der Kruit 2004) might be systematically biased toward too high or low velocities as well. Unless no bar or strong perturbation is shown to exist in their central regions, caution should be taken when modeling these velocity curves because a steep (shallow, respectively) velocity rise could mimic a stellar component that is too (less) concentrated, or a dark matter halo that is too cuspy (shallow; see also Rhee et al. 2004; Valenzuela et al. 2007; Dicaire et al. 2008, for less inclined disk cases).

Acknowledgements. We are grateful to Frédéric Bournaud and Justin Read for insightful discussions. We also thank the anonymous referee for constructive comments and suggestions. L.C. acknowledges financial support from Centre National d'Études Spatiales. F.R. acknowledges support from the European Research Council through the grant ERC-StG-335936.

\section{References}

Asaki, Y., Deguchi, S., Imai, H., et al. 2010, ApJ, 721, 267

Benjamin, R. A., Churchwell, E., Babler, B. L., et al. 2005, ApJ, 630, L149

Bissantz, N., Englmaier, P., \& Gerhard, O. 2003, MNRAS, 340, 949

Blitz, L., \& Spergel, D. N. 1991, ApJ, 370, 205

Bovy, J., Allende Prieto, C., Beers, T. C., et al. 2012, ApJ, 759, 131

Brunthaler, A., Reid, M. J., Menten, K. M., et al. 2009, ApJ, 693, 424

Burton, W. B., \& Gordon, M. A. 1978, A\&A, 63, 7

Chemin, L., Balkowski, C., Cayatte, V., et al. 2006, MNRAS, 366, 812

Chemin, L., Carignan, C., \& Foster, T. 2009, ApJ, 705, 1395

Chemin, L., de Blok, W. J. G., \& Mamon, G. A. 2011, AJ, 142, 109

Clemens, D. P. 1985, ApJ, 295, 422

de Blok, W. J. G., Walter, F., Brinks, E., et al. 2008, AJ, 136, 2648

Dehnen, W., \& Binney, J. 1998, MNRAS, 294, 429

Dicaire, I., Carignan, C., Amram, P., et al. 2008, MNRAS, 385, 553

Eisenstein, D. J., Weinberg, D. H., Agol, E., et al. 2011, AJ, 142, 72

Emsellem, E., Cappellari, M., Peletier, R. F., et al. 2004, MNRAS, 352, 721

Englmaier, P., \& Gerhard, O. 1999, MNRAS, 304, 512

Famaey, B., \& Binney, J. 2005, MNRAS, 363, 603

Fich, M., Blitz, L., \& Stark, A. A. 1989, ApJ, 342, 272

Fux, R. 1999, A\&A, 345, 787

García-Lorenzo, B., Márquez, I., Barrera-Ballesteros, J. K., et al. 2015, A\&A, 573, A59

Gómez, G. C. 2006, AJ, 132, 2376

Gunn, J. E., Knapp, G. R., \& Tremaine, S. D. 1979, AJ, 84, 1181

Hammersley, P. L., Garzón, F., Mahoney, T. J., López-Corredoira, M., \& Torres,

M. A. P. 2000, MNRAS, 317, L45

Honma, M., Nagayama, T., Ando, K., et al. 2012, PASJ, 64, 136

Kalberla, P. M. W. 2003, ApJ, 588, 805

Kalberla, P. M. W., Burton, W. B., Hartmann, D., et al. 2005, A\&A, 440, 775

Kalberla, P. M. W., Dedes, L., Kerp, J., \& Haud, U. 2007, A\&A, 469, 511

Kawata, D., Hunt, J. A. S., Grand, R. J. J., Pasetto, S., \& Cropper, M. 2014, MNRAS, 443, 2757

Kregel, M., \& van der Kruit, P. C. 2004, MNRAS, 352, 787

Levine, E. S., Heiles, C., \& Blitz, L. 2008, ApJ, 679, 1288

Liszt, H. S., \& Burton, W. B. 1980, ApJ, 236, 779

Marasco, A., \& Fraternali, F. 2012, EPJ Web Conf., 19, 8007

McClure-Griffiths, N. M., \& Dickey, J. M. 2007, ApJ, 671, 427

Merrifield, M. R. 1992, AJ, 103, 1552

Nakanishi, H., \& Sofue, Y. 2003, PASJ, 55, 191

Nakanishi, H., \& Sofue, Y. 2006, PASJ, 58, 847

Navarro, J. F., Hayashi, E., Power, C., et al. 2004, MNRAS, 349, 1039

Reid, M. J., Menten, K. M., Zheng, X. W., et al. 2009, ApJ, 700, 137

Reid, M. J., Menten, K. M., Brunthaler, A., et al. 2014, ApJ, 783, 130

Renaud, F., Bournaud, F., Emsellem, E., et al. 2013, MNRAS, 436, 1836

Rhee, G., Valenzuela, O., Klypin, A., Holtzman, J., \& Moorthy, B. 2004, ApJ, 617, 1059

Robin, A. C., Reylé, C., Derrière, S., \& Picaud, S. 2003, A\&A, 409, 523

Rodriguez-Fernandez, N. J., \& Combes, F. 2008, A\&A, 489, 115

Rygl, K. L. J., Brunthaler, A., Sanna, A., et al. 2012, A\&A, 539, A79

Shiozaki, S., Imai, H., Tafoya, D., et al. 2011, PASJ, 63, 1219

Sofue, Y. 2013, PASJ, 65, 118

Sofue, Y., Honma, M., \& Omodaka, T. 2009, PASJ, 61, 227

Swaters, R. A., Sancisi, R., \& van der Hulst, J. M. 1997, ApJ, 491, 140

Valenzuela, O., Rhee, G., Klypin, A., et al. 2007, ApJ, 657, 773

Wegg, C., \& Gerhard, O. 2013, MNRAS, 435, 1874

Xu, Y., Li, J. J., Reid, M. J., et al. 2013, ApJ, 769, 15

Zhang, B., Moscadelli, L., Sato, M., et al. 2014, ApJ, 781, 89 